\title{
DIRECT NUMERICAL SIMULATIONS OF SECONDARY FLOW AND AIR-WATER GAS TRANSFER IN OPEN-CHANNEL TURBULENCE
}

\author{
Ryosuke TERAOKA $^{1}$, Yuji SUGIHARA ${ }^{2}$, Takuya NAKAGAWA ${ }^{3}$ and \\ Nobuhiro MATSUNAGA ${ }^{4}$ \\ ${ }^{1}$ Student Member of JSCE, Graduate Student, Dept. of Earth System Science and Technology, Kyushu University \\ (6-1 Kasuga-koen, Kasuga 816-8580, Japan) \\ E-mail: teraoka@esst.kyushu-u.ac.jp \\ ${ }^{2}$ Member of JSCE, Assoc. Professor, Dept. of Earth System Science and Technology, Kyushu University \\ (6-1 Kasuga-koen, Kasuga 816-8580, Japan) \\ E-mail: sugihara@esst.kyushu-u.ac.jp \\ ${ }^{3}$ Student Member of JSCE, Graduate Student, Dept. of Earth System Science and Technology, Kyushu University \\ (6-1 Kasuga-koen, Kasuga 816-8580, Japan) \\ E-mail: nakagawa@esst.kyushu-u.ac.jp \\ ${ }^{4}$ Fellow Member of JSCE, Professor, Dept. of Earth System Science and Technology, Kyushu University \\ (6-1 Kasuga-koen, Kasuga 816-8580, Japan) \\ E-mail:matunaga@esst.kyushu-u.ac.jp
}

\begin{abstract}
Direct numerical simulations of the secondary flow and the gas transfer in an open-channel flow with the sidewalls are carried out to investigate the effects of Prandtl's second kind of secondary flow on the turbulence dynamics and the gas transfer at the water surface. Turbulent characteristics on the secondary flow such as the velocity-dip phenomena and counter-rotating vortex pairs near the upper and lower corners are verified to be reproducible in comparisons with existing experimental and numerical results. Turbulent structures visualized based on the second invariant of the velocity gradient tensor make it clear that swirling vortex tubes generate near not only the bed but also the sidewalls of the channel. The turbulence dynamics due to such vortex structures may play important roles in driving the surface velocity divergence. The numerical results don't agree quantitatively with the gas transfer velocity by a surface divergence model. It is concluded from the present results that the secondary flow in open channel influences significantly the gas transfer at the water surface.
\end{abstract}

Key Words: open-channel flow, gas transfer, secondary flow, surface divergence, DNS

\section{INTRODUCTION}

Clarifying the gas transfer at the water surface is important for estimating accurately the flux of carbon dioxide between the atmosphere and the ocean, and it leads to a research task to predict the global warming in the future. The gas transfer mechanism is driven by microscopic turbulent eddies near the water surface.

In previous studies, the mechanism has been investigated in open-channel flows, which are one of the simplest flow systems in fluid mechanics and hydraulic engineering. Sanjou and $\mathrm{Nezu}^{1)}$ examined open-channel flows at various water depths to reveal how the intensity of the surface velocity divergence varies with the water depth, and they proposed a modified surface divergence model for the gas transfer velocity. However, turbulent flow and dissolved-gas concentration fields near the water surface become unsteady and microscopic, so it is hard to measure these quantities accurately. It may be difficult to understand the gas transfer phenomena with microscopic temporal and spatial scales by experimental approaches only.

In recent years, with the development of computer technology, interfacial scalar transfers in openchannel flows have been investigated in terms of numerical simulations. Nagaosa and Handler ${ }^{2}$ carried out direct numerical simulations of open-channel flows at various Reynolds numbers and investigated in detail the time scale of the turbulence controlling scalar transfer at a free surface. However, in previous studies, because of calculation load, periodic conditions were generally imposed on boundaries in spanwise direction. In actual open-channel flows, Prandtl's second kind of secondary flow is driven due to the anisotropy of turbulence occurred by the pres- 


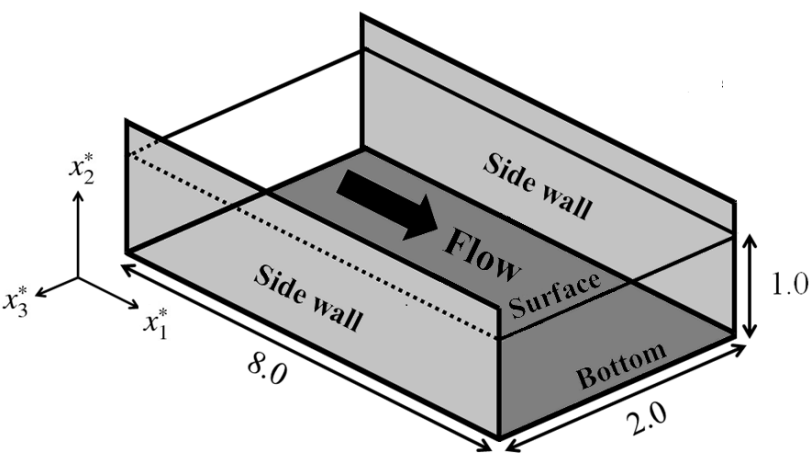

Fig.1 Numerical domain

ence of the sidewalls. Such a secondary flow seems to affect the surface-renewal motions and the gas transfer at the water surface.

As examples of numerical studies in open-channel flows with the sidewalls, Hayashi et al. ${ }^{3)}$ carried out a direct numerical simulation at channel aspect ratio of 2, and revealed the formation and preservation mechanisms of the secondary flow on the basis of the Reynolds momentum equations. Joung and $\mathrm{Choi}^{4)}$ also made a direct numerical simulation at channel aspect ratio of 1 . They examined dominant coherent structures near the sidewalls by using the Quadrant analysis. Direct numerical simulations of openchannel flows with the sidewalls have been performed until now, but researches on the gas transfer have hardly been conducted, though focusing on the effects of the secondary flow is important in understanding the environment in river.

The present study is intended to investigate the effects of the secondary flow on the turbulence dynamics and the gas transfer mechanism in an open channel flow. We carry out a direct numerical simulation of an open-channel flow with the sidewalls. Since Chan and Scriven ${ }^{5)}$ and Awaya and $\mathrm{Abe}^{6}$ ) have ever reported techniques to quantify the gas transfer velocity using the surface velocity divergence, we also examine whether the techniques are effective or not for the present results.

\section{NUMERICAL SIMULATION}

In the present study, we carry out a direct numerical simulation (hereafter DNS) of an open-channel flow with the sidewalls at channel aspect ratio of 2 . Fig.1 shows a schematic diagram of the numerical domain. A computational code in this study is based on a opened DNS code using the simplified MAC method by Kajishima ${ }^{7}$, and the details of the numerical method was also reported in Sugihara et al. ${ }^{8)}$. The basic equations include the continuity equation, the
Table1 Numerical conditions

\begin{tabular}{|l|c|}
\hline $\begin{array}{l}\text { Size of compu- } \\
\text { tational domain }\end{array}$ & \multicolumn{2}{|l|}{$\begin{array}{l}\text { Streamwise } \\
\text { Interfacial-normal } x_{2}^{*}: 1.0 \\
\text { Spanwise }\end{array}$} \\
\hline Number of grid & $\begin{array}{r}\left(N_{x 1}, N_{x 2}, N_{x 3}\right) \\
=(128,129,129)\end{array}$ \\
\hline Grid spacing & $\Delta x_{1}^{+}: 9.375$ \\
(in wall unit) & $\Delta x_{2}^{+}: 0.3 \sim 2.25$ \\
& $\Delta x_{3}^{+}: 0.45 \sim 4.5$ \\
\hline Time difference & $\Delta t^{*}=2.0 \times 10^{-5}$ \\
\hline Total times & $T^{*}=25$ \\
\hline Parameters & $\operatorname{Re}_{\tau}=150, S c=1, F r_{\tau}=1 / \sqrt{2}$ \\
\hline
\end{tabular}

Navier-Stokes equations and the advection-diffusion equation of the dissolved gas.

Using the friction velocity averaged over the bed and the sidewalls $u_{\tau}$, the depth of water $H$, the density $\rho$ and the concentrations at the water surface $c_{s}$ and at the bed $c_{b}$, these equations are nondimensionalized according to the following relations:

$$
\left.\begin{array}{c}
u_{i}=u_{\tau} u_{i}^{*}, x_{i}=H x_{i}^{*}, p=\rho u_{\tau}^{2} p^{*} \\
t=\frac{H}{u_{\tau}} t^{*}, c-c_{b}=\left(c_{s}-c_{b}\right) c^{*}
\end{array}\right\}
$$

where $*$ denotes the dimensionless quantity. By using these dimensionless quantities, the basic equations are described as follows:

$$
\begin{gathered}
\frac{\partial u_{i}^{*}}{\partial x_{i}^{*}}=0 \\
\frac{\partial u_{i}^{*}}{\partial t^{*}}+u_{j}^{*} \frac{\partial u_{i}^{*}}{\partial x_{j}^{*}}=-\frac{\partial p^{*}}{\partial x_{i}^{*}}+\frac{1}{R e_{\tau}}\left(\frac{\partial^{2} u_{i}^{*}}{\partial x_{j}^{*} \partial x_{j}^{*}}\right)+\frac{\delta_{1 i}}{F r_{\tau}^{2}} \\
\frac{\partial c^{*}}{\partial t^{*}}+u_{j}^{*} \frac{\partial c^{*}}{\partial x_{j}^{*}}=\frac{1}{S c R e_{\tau}}\left(\frac{\partial^{2} c^{*}}{\partial x_{j}^{*} \partial x_{j}^{*}}\right)
\end{gathered}
$$

where dimensionless parameters providing the state of the flow are Reynolds number $R e_{\tau}$, Froude number $F r_{\tau}$ and Schmidt number $S c$, and they are defined by

$$
R e_{\tau}=\frac{u_{\tau} H}{v}, F r_{\tau}=\frac{u_{\tau}}{\sqrt{g H \sin \theta}}, S c=\frac{v}{D}
$$

where $v$ is the kinematic viscosity, $\sin \theta$ the channel bed slope, and $D$ the molecular diffusivity of the dissolved gas.

In this numerical simulation, we set $R e_{\tau}=150$ and $F r_{\tau}=1 / \sqrt{2}$ at which a uniform flow is established by the balance between the averaged frictional and the gravitational forces. Also, $S c=1$ is given because of the limitation of calculation load. This value is much smaller than those of carbon dioxide $(S c=600)$ and oxygen $(S c=480)$, but we consider the dynamics controlling the gas transfer to be similar between both.

We impose the periodic boundary conditions on 


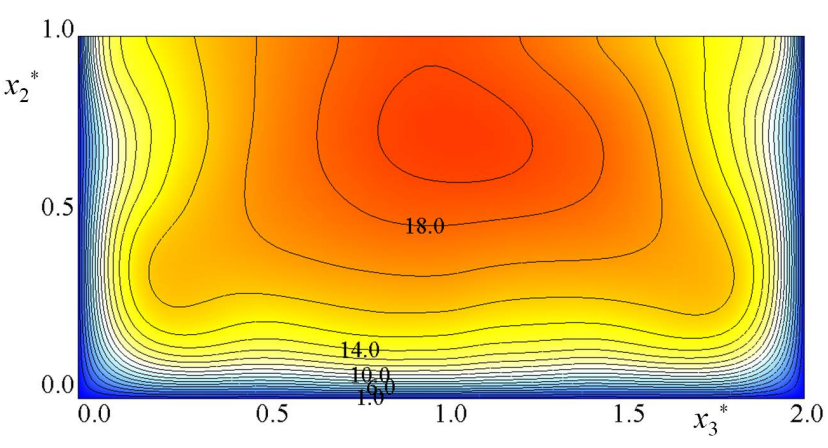

Fig.2 Contour map of averaged stremwise velocity in cross section

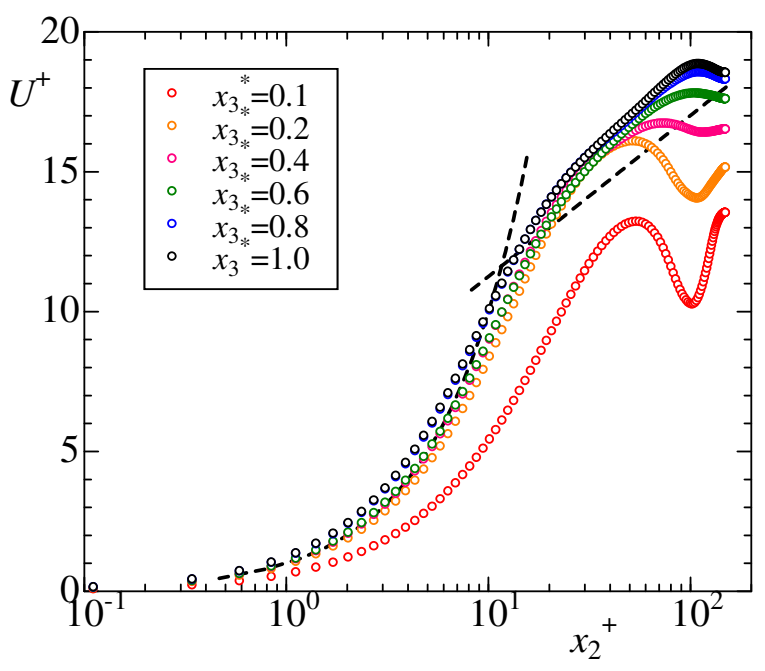

Fig.3 Vertical profiles of averaged stremwise velocity

the velocities in streamwise direction, and also give the velocity conditions on the bed, the water surface, and the sidewalls as follows:

- For the velocities on the bed and the water surface

$$
\left.\begin{array}{r}
u_{1}^{*}=u_{2}^{*}=u_{3}^{*}=0 \quad \text { at } x_{2}^{*}=0 \\
\frac{\partial u_{1}^{*}}{\partial x_{2}^{*}}=\frac{\partial u_{3}^{*}}{\partial x_{2}^{*}}=0, u_{2}^{*}=0 \quad \text { at } x_{2}^{*}=1
\end{array}\right\}
$$

- For the velocities on the sidewalls

$$
u_{1}^{*}=u_{2}^{*}=u_{3}^{*}=0 \quad \text { at } x_{3}^{*}=0,2
$$

For the dissolved gas, we impose the periodic boundary conditions on the boundaries in streamwise direction, and the following boundary conditions are given on the bed, the water surface, and the sidewalls:

- For the dissolved gas on the bed and the water surface

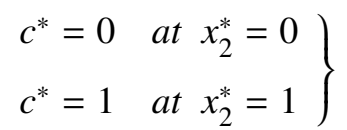

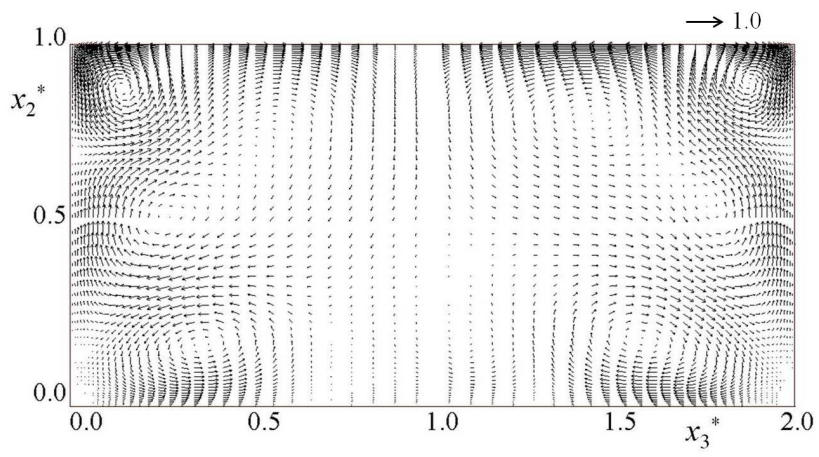

Fig.4 Averaged secondary-flow vectors in cross section

- For the dissolved gas on the sidewalls

$$
\frac{\partial c^{*}}{\partial x_{3}^{*}}=0 \quad \text { at } x_{3}^{*}=0,2
$$

As boundary conditions of the pressure, the periodic and the Neumann conditions are imposed in streamwise direction, and on the bed and the sidewalls, respectively.

The governing equations are integrated under the condition of the dimensionless time step of $2.0 \times 10^{-5}$, and we analyze the numerical data after dimensionless time of 25 at which the statistically steady turbulence has been established. The details of the numerical conditions are summarized in Table 1, where + shows dimensionless quantity based on the wall-layer scaling using the averaged wall friction velocity $u_{\tau}$ and the kinematic viscosity $v$.

\section{RESULTS AND DISCUSSION}

\section{(1) Turbulent structures}

In previous studies, direct numerical simulations have not been carried out for the gas transfer in openchannel flows with sidewalls. First, we compare turbulent characteristic quantities obtained from the present numerical simulation with previous experimental and numerical results to confirm the validity of the reproduced turbulent field. This is also important to make sure the accuracy of the numerical results for the gas transfer. It should be noted that the dimensionless quantities based on Eq.(1) are shown in the following figures.

Fig.2 shows a cross-section contour map of the averaged streamwise velocity $U^{+}$. This figure shows the maximum streamwise velocity appears not at the water surface, but at depth of $x_{2}^{*}=0.7 \sim 0.8$. The numerical results reproduce the velocity-dip phenomena which can be observed in an open-channel of low aspect ratio (less than 5).

Fig.3 shows vertical profiles of the averaged streamwise velocity $U^{+}$in six cross sections in span- 


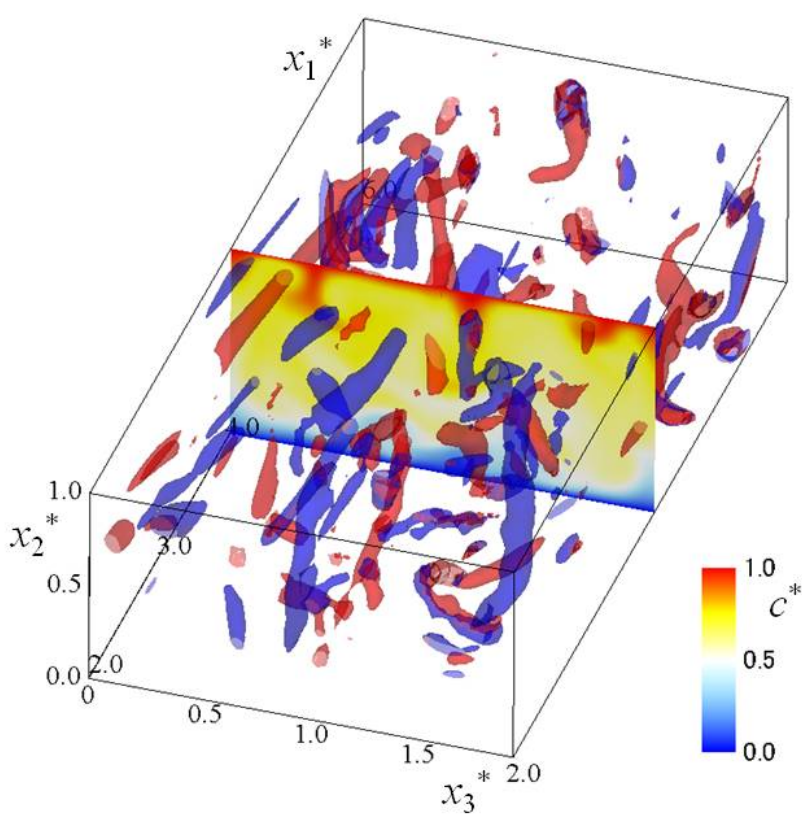

Fig.5 Swirling vortex tubes and dissolved-gas concentration in a cross section $x_{1}^{*}=4.0$

wise direction. The dotted lines in the figure indicate the expressions of $U^{+}=x_{2}^{+}$and $U^{+}=2.5 \ln x_{2}^{+}+5.5$, respectively. The numerical results rules the relation $U^{+}=x_{2}^{+}$near the bed, and show the velocity-dip phenomena in all the sections. The results also almost agree with the logarithmic velocity distribution in a region far from the bed excepting at $x_{3}^{*}=0.1$ near the sidewalls. It is concluded from these distributions that the secondary flow has significant influence on the averaged streamwise velocity in the simulation of low aspect ratio. The behavior of the streamwise velocity are similar to the numerical results obtained from Hayashi et al. ${ }^{3)}$ and Joung and Choi ${ }^{4}$.

Fig.4 shows a cross-section distribution of the averaged secondary-flow vectors. Counter-rotating vortex pairs are observed near the upper and lower corners in the channel. Three kinds of vortex structures, i.e., the bottom vortex, the surface vortex and the inner secondary flow, can be reproduced, and these structures are driven by the turbulence anisotropy. The value of the maximum secondary flow velocity takes approximately 3 percent of the maximum streamwise velocity, and it is supported by experimental and numerical values, which were reported in previous studies. In particular, Nezu et al. ${ }^{9}$ revealed the location of the bottom vortex under conditions of various channel aspect ratios, and our results provide the similar results to their ones.

In open-channel flows, surface-renewal motions and scalar transfers on the water surface are affected by the collapsing of swirling vortex tubes, which are

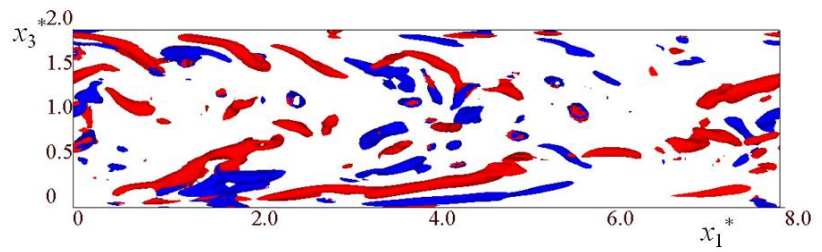

(a) $x_{1}^{*}-x_{3}^{*}$ cross-section view (near the water surface: $x_{2}^{*}=0.8 \sim 1.0$ )

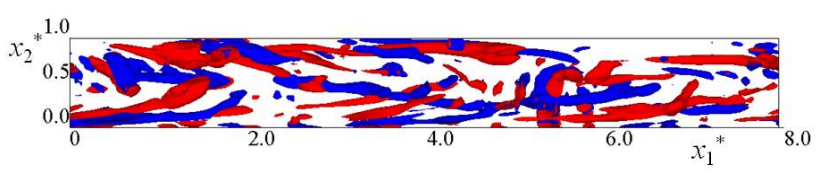

(b) $x_{1}^{*}-x_{2}^{*}$ cross-section view (near the sidewalls: $\left.x_{3}^{*}=0.0 \sim 0.3\right)$

Fig.6 Visualized swirling vortex tube's structures

originally generated near the bed and the sidewalls. The vortex tubes are defined as a region that the second invariant of the velocity gradient tensor $Q^{*}$ becomes positive, and $Q^{*}$ is expressed by

$$
Q^{*}=\frac{1}{2}\left(u_{i, j}^{* 2}-u_{i, j}^{*} u_{j, i}^{*}\right)=\frac{1}{2}\left(\left\|\Omega^{*}\right\|^{2}-\left\|S^{*}\right\|^{2}\right)
$$

where

$$
S_{i j}^{*}=\frac{1}{2}\left(\frac{\partial u_{i}^{*}}{\partial x_{j}^{*}}+\frac{\partial u_{j}^{*}}{\partial x_{i}^{*}}\right), \quad \Omega_{i j}^{*}=\frac{1}{2}\left(\frac{\partial u_{i}^{*}}{\partial x_{j}^{*}}-\frac{\partial u_{j}^{*}}{\partial x_{i}^{*}}\right)
$$

$S_{i j}^{*}$ is the strain rate tensor, $\Omega_{i j}^{*}$ the vortex tensor, and $\left\|\Omega^{*}\right\|$ and $\left\|S^{*}\right\|$ the Frobenius norms of the strain rate tensor and the vortex tensor, respectively. The velocity shear becomes smaller as the water surface approaches, so that $Q^{*}$ should be normalized by the root-mean-square of $Q^{*}$ at each depth to visualize distinctly the swirling vortex tubes over the whole depth. The value of $Q^{*}$ in this study also includes information on the direction of rotation by using the sign of the streamwise vorticity $\omega_{1}^{*}$.

Fig.5 shows the swirling vortex tube's structure visualized based on the $Q$ method and the dissolved-gas concentration, where the visualized vortex tubes and the dissolved-gas concentration are drawn in the region of $x_{1}^{*}=2.0 \sim 6.0$ and in the $x_{2}^{*}-x_{3}^{*}$ cross-section at $x_{1}^{*}=4$, respectively. Figs.6(a) and (b) also show the vortex structures in the $x_{1}^{*}-x_{3}^{*}$ cross section near the water $\operatorname{surface}\left(x_{2}^{*}=0.8 \sim 1.0\right)$ and in the $x_{1}^{*}-x_{2}^{*}$ cross section near the sidewall $\left(x_{3}^{*}=0.0 \sim 0.3\right)$, respectively. The clockwise and anticlockwise rotating vorticities toward streamwise direction are denoted by the colors of red and blue, respectively. From these figures, we can observe the vortex tubes to generate near the not only the bed but also the sidewalls. It is conceivable that these vortex tubes are stretched in streamwise 


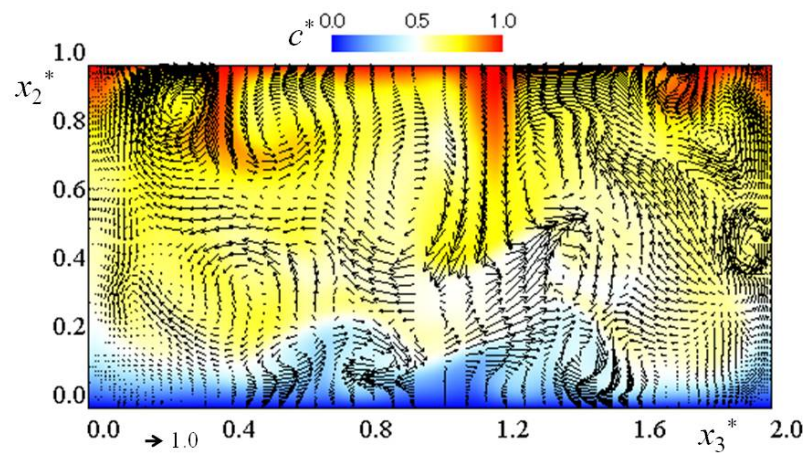

Fig.7 Cross-section distributions of transient secondaryflow vectors and dissolved-gas concentration

direction and are broken. The turbulence generated by such processes is advected near the water surface. The swirling vortex tubes from the sidewalls may be considered as coherent structures concerned with the secondary flow, and the structures from both sidewalls are seen to be merged around the center of the channel. This suggests that the interaction between the turbulence structures from both sidewalls acts on the surface-renewal motions. These results are similar to existing numerical results and support the validity of the present simulation.

\section{(2) Relationship between fields of turbulence and dissolved-gas concentration}

In the previous section, we confirmed that the present results about the turbulence field obtained from the DNS are similar to experimental and numerical results reported before. We examine the relation between the turbulence and the dissolved-gas concentration. Fig.5 shows the swirling vortex tubes to influence obviously the dissolved-gas concentration near water surface, and the concentration boundary layer fluctuates violently due to the vortex structures.

Fig.7 shows distributions of the velocity vectors and the concentration of the dissolved gas in the $x_{2}^{*}-x_{3}^{*}$ cross-section at $x_{1}^{*}=4$ as shown in Fig.5. The instantaneous inner secondary flows are observed around $x_{3}^{*}=0.2$, where the concentration boundary layer gets thin in upwelling flow area and becomes thicker in downward flow area under the influence of the secondary flows. The local vertical gradient of the concentration is larger in upwelling area, so that the gas flux increases significantly there. Furthermore, a downward flow exists around $x_{3}^{*}=1.1$. The flow structure may induce the velocity-dip phenomena in the center of open channel and it thickens the concentration boundary layer.
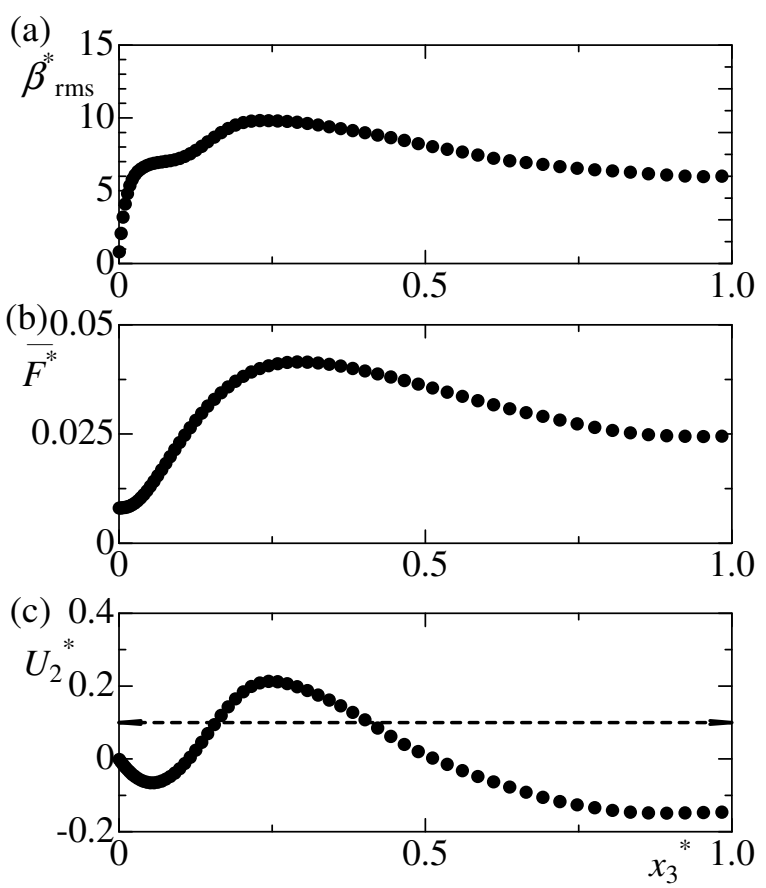

Fig.8 Cross-section line distributions of (a) root-meansquare of surface velocity divergence, (b) averaged gas flux, and (c) averaged vertical velocity due to secondary flow at $x_{2}^{*}=0.7$, which shown in the region of $x_{3}^{*}=0.0 \sim 1.0$

\section{(3) Relationship between turbulence and gas flux on water surface}

Some previous studies reported that the surface velocity divergence becomes an important physical quantity to quantify the gas transfer velocity ${ }^{10), 11)}$. Thus, we attempt to check the relationship between the surface divergence and the gas flux in the present results. The surface divergence $\beta^{*}$ is a physical quantity to describe divergent and convergent motions of the water surface, and it is defined as

$$
\beta^{*}=\left.\left(\frac{\partial u_{1}^{*}}{\partial x_{1}^{*}}+\frac{\partial u_{3}^{*}}{\partial x_{3}^{*}}\right)\right|_{\text {surface }}
$$

The gas flux $F^{*}$ is also given by

$$
F^{*}=\left.\frac{1}{R e_{\tau} S c} \frac{\partial c^{*}}{\partial x_{2}^{*}}\right|_{\text {surface }}
$$

where the flux is defined to be positive in downward direction. Figs.8(a) (c) show the cross-section line distributions of the root-mean-square of the surface velocity divergence $\beta_{r m s}^{*}$, the averaged interfacial gas flux $\overline{F^{*}}$, and the averaged vertical velocity $U_{2}^{*}$ at $x_{2}^{*}=0.7$, respectively. They are shown in the region of $x_{3}^{*}=0.0 \sim 1.0$. From these figures, we can confirm that these physical quantities take the maximum value around $x_{3}^{*}=0.25$. The point at which they take the maximum value agrees with the location where 


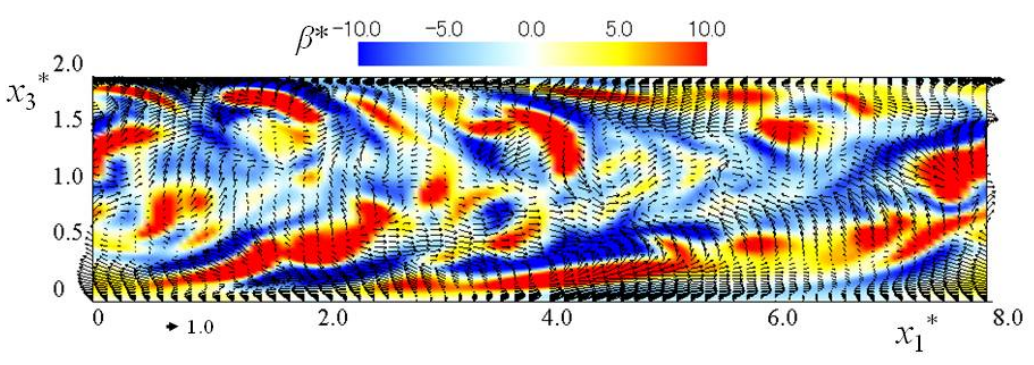

(a)Surface velocity divergence

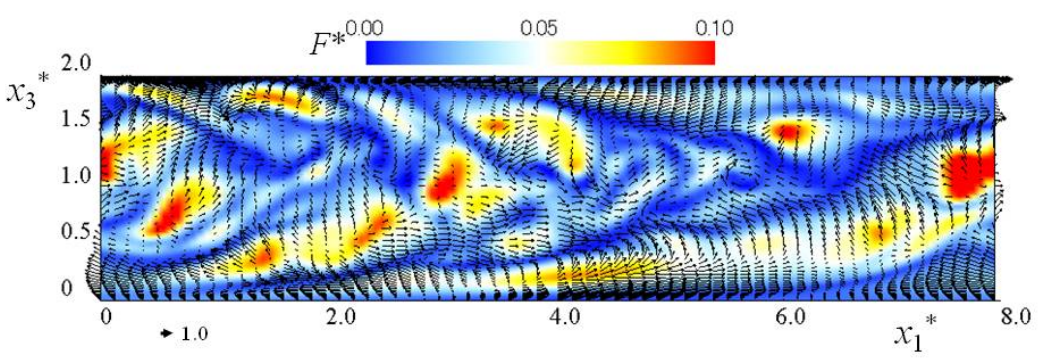

(b)Gas flux on water surface

Fig.9 Spatial distributions of surface velocity divergence and gas flux on water surface in open channel with sidewalls

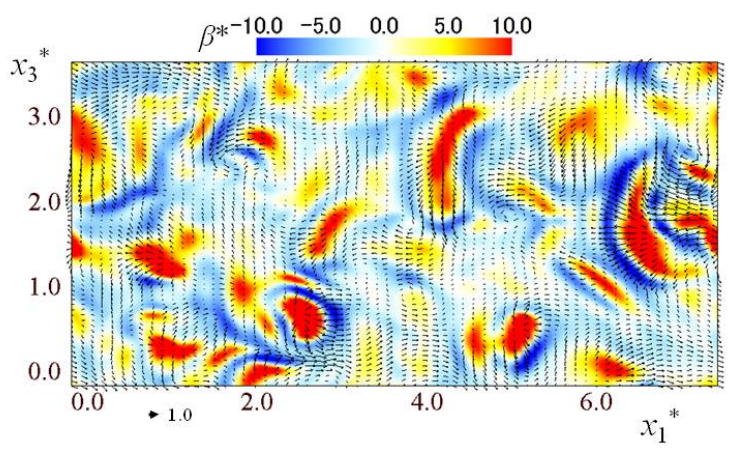

(a)Surface velocity divergence

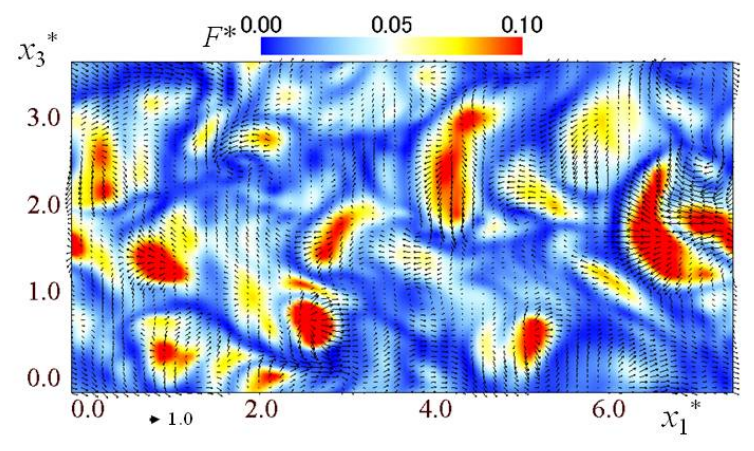

(b)Gas flux on water surface

Fig.10 Spatial distributions of surface velocity divergence and gas flux on water surface in open channel without sidewalls

the upward current occurs due to the inner secondary flows near the upper corner in the channel. Thus, we can see that the surface velocity divergence and the interfacial gas flux are obviously affected by the secondary flows from the sidewalls.

Figs.9(a) and (b) show spatial distributions of the surface divergence and the gas flux on the water surface, respectively. For comparison, we also show spatial distributions of the surface divergence and the gas flux in the case without the sidewalls in Figs.10(a) and (b). Fig.9(a) demonstrates peculiar positive surface divergence regions, which become oblique beltshaped, to be similar to the spatial pattern of the swirling vortex tube's structures near the sidewalls, and they generate alternately from each sidewall. The belt-shaped regions are stretched from the sidewalls to the channel center. Around the central region, the belt-shaped structures merge and the negative divergence region appears to be dominant. On the other hand, in the open-channel flow without the sidewalls, the positive surface divergence region occurs patchwise as shown in Fig.10(a). These results support the surface divergence structure to be significantly affected by the secondary flows generated from the sidewalls. With respect to the gas flux on the water surface, the downward gas flux regions agree qualitatively with the positive surface divergence regions in the flows both with and without the sidewalls. This indicates that upwelling currents lift up low concentration fluid from the bulk region, and the divergent motion stretches horizontally the concentration boundary layer, hence the vertical concentration gradient increases locally. The region of the positive surface divergence is affected by the secondary flows, so that 
(a)

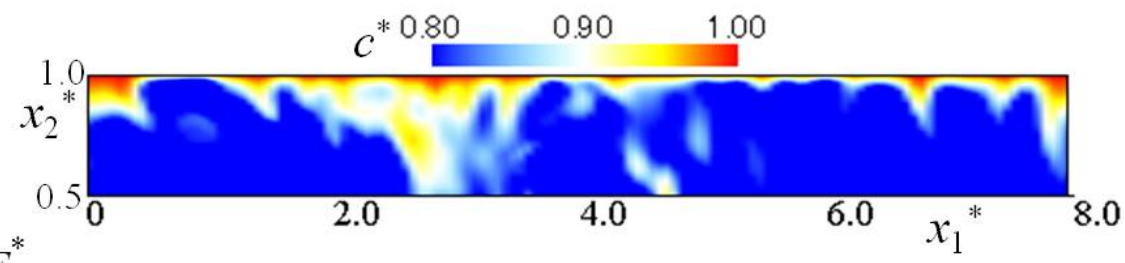

(b) $F^{*}$.
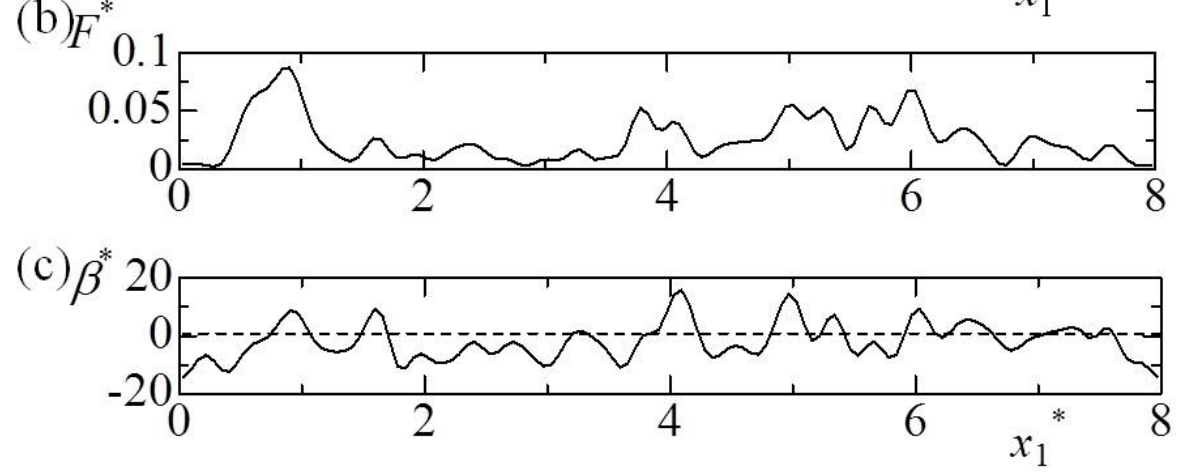

Fig.11 Spatial distributions along center line of channel for (a) concentration boundary layer, (b) gas flux, and (c) surface velocity divergence on water surface

they have significant influences on the gas transfer.

Fig.11(a) shows vertical structures of the dissolved gas concentration along the center line of the channel, and Fig.11(b) and (c) shows horizontal profiles of the gas flux and the surface divergence to examine their relationship. It should be noticed in Fig.11(a) that the region of $x_{2}^{*}=0.5 \sim 1.0$ is demonstrated and enlarged twice in vertical scale. From these figures, the thickness of the concentration boundary layer fluctuates widely, and the gas flux increases in the positive surface divergence region. This is because the dissolved gas is transported horizontally there, and the concentration boundary layer becomes thinner in such a region. In the negative surface divergence region in which high concentration fluid is transported to the bulk region, the concentration boundary layer seems to be relatively thicker, and the interfacial gas flux decreases there. As shown in Fig.10, the positive surface divergence obtained from the present numerical results under the influence of the secondary flow is larger in comparison with the case without the secondary flow, whereas the gas flux may appear to be smaller despite the large value of the surface divergence. Thus, the quantitative relationship between both is different from that of the case without the sidewalls.

\section{(4) Comparison of gas transfer velocity by sur- face divergence model with numerical results}

The gas transfer velocity close to the flow stagnation is examined under the assumption of the water surface with no fluctuation. At the flow stagnation, the vertical advection becomes dominant, and it is

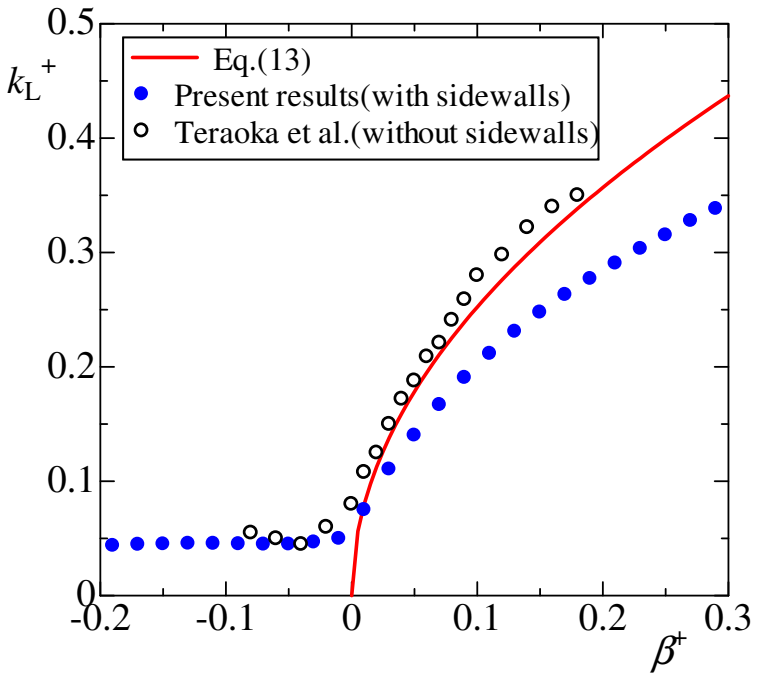

Fig.12 Comparison between local gas transfer velocity model and numerical results

balanced by the vertical diffusion. This situation is called the stagnation flow approximation ${ }^{5)}$. The exact solution can be obtained from the advection-diffusion equation under this approximation as follows:

$$
k_{L}^{+}=\sqrt{\frac{2}{\pi}} S c^{-1 / 2} \sqrt{\beta^{+}}
$$

where + shows the wall-layer scaling dimensionless quantity. The above relation is considered as a typical surface divergence model. Fig.12 shows the local gas transfer velocity in this study, which is calculated by the moving-average at infinitesimal interval $\Delta \beta^{+}$. In the figure, Eq.(13) denoted by the red line is shown to compare the gas transfer velocity from the present 
study with the surface divergence model. In addition, the results from the DNS without the sidewalls are also demonstrated by the circle ${ }^{12}$. We should note that Eq.(13) can be realized in only the positive divergence region, whereas the present results is free from such a limitation. This figure shows the gas transfer velocity takes a constant value approximately for the negative divergence. On the other hand, for the positive divergence, the local gas transfer velocity increases monotonically with increase of the local surface divergence. However, the present results is quantitatively different from the results in the case without the sidewalls and the model solution of Eq.(13). It can be concluded from the quantitative comparison that the presence of the secondary flow gives significant influences on the gas transfer at the water surface.

\section{CONCLUDING REMARKS}

In this study, we carried out the DNS of the gas transfer in the open-channel flow with the sidewalls to investigate how the secondary flow affects the turbulence dynamics and the air-water gas transfer, and examined the gas transfer mechanism in comparison with the surface divergence model. The present numerical results reproduce the velocity-dip phenomena that the maximum streamwise velocity appears under the water surface. The counter-rotating vortex pairs are also observed near the upper and lower corners in the channel. These characteristics agree with those well-known in open-channel flows with the sidewalls. The visualization based on the $Q$ method shows that the swirling vortex tubes may affect significantly the surface-renewal motions. The surface divergence structure is also influenced by the secondary flow and the pattern is different from the results obtained from the DNS without the sidewalls. The positive surface divergence region corresponds to the downward gas flux region qualitatively, where the swirling vortex tubes exist intensively, and the secondary flow may influence on the surface gas transport. The comparison between the surface divergence model and the present results shows that both behaviors don't agree and the turbulent mechanism dominated by the secondary flow has influence on the gas transfer at the water surface.

We are planning to carry out DNS at various Reynolds numbers and establish a physical model describing the relationship between the secondary flow and the gas transfer.
ACKNOWLEDGMENT: The authors thank Prof. K. Shiono at Loughborough University for helpful discussions. This work was partially supported by a Grant-in-Aid for Scientific Research from the Japan Society for the Promotion of Science.

\section{REFERENCES}

1) Sanjou, M. and Nezu, I.: Experimental study on surface velocity divergence and interfacial gas transfer in open-channel flows, Journal of JSCE, Vol.1, pp.82-89, 2013.

2) Nagaosa, R. and Handler, R. A.: Characteristic time scales for predicting the scalar flux at a free surface in turbulent open-channel flows AIChE Journal Vol.58, No.12, pp.3867-3877, 2012.

3) Hayashi, S., Ohmoto, T. and Honda, I.: Direct Numerical simulation of secondary currents and vortex structures in an open channel flow Journal of Japan Society of Civil Engineers Ser. B, Vol.62, No.1, pp.8099, 2006 (in Japanese).

4) Joung, Y. and Choi, S.: Direct Numerical simulation of low Reynolds number flows in an open-channel with side walls Int. J. Numer. Meth. Fluids, Vol.62, pp.854-874, 2009.

5) Chan, W. C. and Scriven, L. E.: Absorption into irrotational stagnation flow - a case study in convective diffusion theory Ind. Eng. Chem. Fund., Vol.9(1), pp.114-120, 1970.

6) Awaya, Y. and Abe, T.:On turbulent characteristics and aeration rate at the water surface(II), The 26th Japan Society of Civil Engineers Congress II, pp.491492, 1971(in Japanese).

7) Kajishima, T.: Numerical Simulation of Turbulent Flows, Yokendo Ltd., 2003 (in Japanese).

8) Sugihara, Y. Nakagawa, D. and Shiono, K.: Direct numerical simulation of gas transfer at the air-water interface, Journal of Japan Society of Civil Engineers, Ser. B2 (Coastal Engineering), Vol.67, No.2, I_071I_075, 2011 (in Japanese).

9) Nezu, I. and Nakagawa, H.: Experimental study on tree-dimensional flow in straight channel, Journal of Japan Society of Civil Engineers, Vol.29, pp.769-774, 1985 (in Japanese).

10) McCready, M. J., Vassiliadou, E. and Hanratty, T. J.: Computer simulation of turbulent mass transfer at a mobile interface, AIChE J. ,Vol.32, pp.1108-1115, 1986.

11) Banerjee, S., Lakehal, D. and Fulgosi, M.: Surface divergence models for scalar exchange between turbulent streams, Int. J. Multiphase Flow, Vol.30, 963-977, 2004.

12) Teraoka, R., Sugihara, Y., Nakagawa, D. and Shiono, K.: Direct numerical simulation of gas transfer at wind-sheared air-water interface, Journal of Japan Society of Civil Engineers, Ser. B2(Coastal Engineering), Vol.70, No.2, I_46-I_50, 2014 (in Japanese).

(Received June 23, 2015) 